\title{
Hepatitis $C$ treatment in drug users: an approach in terms of evidence-based medicine
}

\author{
George Floros*1, Vassiliki Anagnostou², Fotini Ferenidou², \\ Grigorios Lavrentiadis ${ }^{1}$, George Garyfallos ${ }^{1}$ and Ioannis Giouzepas ${ }^{1}$
}

\author{
Address: ${ }^{2}$ nd Department of Psychiatry, School of Medicine, Aristotle University of Thessaloniki, Greece and ${ }^{2}$ School of Medicine, Aristotle \\ University of Thessaloniki, Greece \\ * Corresponding author
}

from International Society on Brain and Behaviour: 3rd International Congress on Brain and Behaviour

Thessaloniki, Greece. 28 November - 2 December 2007

Published: 17 April 2008

Annals of General Psychiatry 2008, 7(Suppl I):S249 doi:I0.I 186/I744-859X-7-SI-S249

This abstract is available from: http://www.annals-general-psychiatry.com/content/7/SI/S249

(c) 2008 Floros et al.; licensee BioMed Central Ltd.

\section{Background}

Recently a consensus was reached on the treatment of the HCV-infected, injection drug user (IDU) with pegylated interferon and ribavirin [1]. It is yet unclear as to whether these best-practice guidelines have been followed to good result due to multiple perceived barriers to treatment initiation.

\section{Materials and methods}

A systematic literature search in PubMed / MEDLINE, was performed. Peer-reviewed journal articles investigating barriers to treatment and therapeutic outcomes were retrieved and critically appraised. Twenty studies identifying issues related to treatment initiation and twenty-six studies presenting therapeutic outcomes were analyzed. Results are summarized and presented in evidence tables. Pooling and further statistical analysis was not justified because of heterogeneity of the identified studies.

\section{Results}

Despite being handicapped by negative views on treatment eligibility, clinical studies indicate that IDUs can be successfully treated for HCV, even in the setting of ongoing drug use. Rates of sustained virological response (SVR) were similar to control groups while patient compliance, treatment adverse effects and potential re-infection didn't have a significant impact on the therapeutic outcome. Therapy can be initiated during the early phase of drug treatment, regardless of the drug treatment strategy.

\section{Conclusions}

The evolving body of medical evidence indicates that IDUs can be effectively treated for HCV despite the persistence of putative, psychosocial barriers to treatment. Our approach to an addicted patient who needs HCV treatment, should not be clouded by perception or prejudice, but instead focus on patient education, interdisciplinary collaboration and close monitoring of the appropriate biological treatment.

\section{References}

I. Crone C., Gabriel G.M: Comprehensive review of hepatitis C for psychiatrists: risks, screening, diagnosis, treatment, and interferon-based therapy complications. Journal of psychiatric practice $2003,9(2): 93-110$. 